\title{
The Development of Lithium Ion Recovery Method by Activated Garbon and Natural Zeolite-based Adsorbent
}

\author{
Chandra Wahyu Purnomo *,1 \\ Endhy Kesuma ${ }^{1}$ \\ Sang Kompiang Wirawan ${ }^{1}$ \\ Hirofumi Hinode ${ }^{2}$ \\ 1 Chemical Engineering Department, Engineering Faculty, Gadjah Mada University, \\ Indonesia \\ 2 Transdiciplinary Science and Engineering Department, Tokyo Institute of Technology, \\ Japan \\ *e-mail: chandra.purnomo@ugm.ac.id
}

Coconut shell char and natural zeolite been activated and then tested to separate between lithium and cobalt ions in solutions. The capacity of adsorption and selectivity between the ions were investigated. The longer adsorption time, the capacity was getting better but the selectivity decreased. It was observed that the optimum selectivity was obtained by 3 hours adsorption using activated carbon. The chemical activated sample has superior performance and selectivity compared with physical activation carbons of the same activation temperature. After the adsorption, pure lithium solution can be obtained while the other metal is adsorbed inside the adsorbent.

Keywords: selective adsorption, activated carbon, natural zeolite, lithium recovery

\section{INTRODUCTION}

Recovery of lithium is become increasingly important recently since the increase of usage of lithium based battery in many modern applications. During the early metal recovery stage from spent battery by hydrometallurgy usually the cathodes will be diluted using strong acid solution to obtain mixture of metal ions mostly contain $\mathrm{Li}, \mathrm{Co}, \mathrm{Ni}, \mathrm{Mn}$, and several others depend on the battery type. After the dilution the next effort is to separate each of ion independently such as by multistage precipitation, electro dialysis (ED), adsorption and other methods (A. Tuncuk et al., 2012).

The precipitation method requires many chemicals to adjust the solubility of each component properly and it consist of several steps of mixing, evaporating and filtering. Meanwhile, ED incorporates membrane and electric current to separate among the ions, however the technology is still expensive and prone to membrane fouling. In this study a simple method of 
92 The Development of Lithium Ion Recovery Method by Activated Carbon and Natural Zeolitebased Adsorbent

separating lithium from the other ion was done by adsorption. The key of the process lays on the usage of adsorbent which can separate selectively the lithium and other metals. For simplicity the adsorption was using synthetic leaching solution only contain lithium and cobalt as the most contained metals on the recent commercial battery.

Adsorption of lithium has been widely investigated and the focus was to produce adsorbent which can adsorp lithium as much as possible. Most of the previous study is using pure lithium solution without any other metals ion for developing the adsorbent (J. M. Jeong et al., 2015). Lithium is a light element which is quite difficult to be adsorp compared with other possible contained metals in the battery leaching solution since the interaction between the lithium ion and the surface is the weakest. To enhance the uptake of lithium some studies use expensive materials as the adsorbent (J. Lemaire et al., 2012; J. L. Xiao et al., 2015). Moreover, it should be aware that the strong adsorbant for lithium proved by adsorption in the lithium solution only may also has strong affinity to the other metals in the real ion mixture solution thus the separation effect will not be obtained.

In this study, low cost adsorbents i.e. activated char and activated natural zeolite were expected to be able to take strongly other metal ions (cobalt) while remain low attraction to the lithium ion. The adjustment of the selectivity is only by varying the activation method and activation temperature for creating a unique surface property. The method will produce pure lithium solution from the leachate mixture than can be precipitated easily after the separation. The other metal ions will be contained inside the adsorbents for further recovery.

\section{EXPERIMENTAL}

\section{Activated Carbon Preparation}

Coconut shell charcoal was sieved to obtain 4-6 mesh particles then activated by $\mathrm{CO}_{2}$ physical activation method. The activation temperature variation were 800 and $900{ }^{\circ} \mathrm{C}$ with temperature increasing rate of $20^{\circ} \mathrm{C} / \mathrm{min}$ and gas flow rate of 2 $\mathrm{ml} / \mathrm{min}$. Activated char then washed with distilled water to remove the debris or powder created from the previous stages that may interfere the adsorption result, then dried in an oven.

Other part of the prepared coconut shell charcoal was chemically activated using $\mathrm{KOH} 10 \% \mathrm{w} / \mathrm{w}$. Charcoal that has been impregnated with $\mathrm{KOH}$ solution then activated using horizontal furnace at two temperature variation of 700 and $750{ }^{\circ} \mathrm{C}$ under nitrogen flow at $2 \mathrm{ml} / \mathrm{min}$. The holding time at the activation temperature was 1 or 2 hours. During the holding time in several activations, $\mathrm{CO}_{2}$ gas was flowed in the flow rate of $2 \mathrm{ml} / \mathrm{min}$. Activated carbon then washed with distilled water for several times until the $\mathrm{pH}$ of spent washing liquid around 7.0, then dried in an oven. All the sample is coded and listed in Table 1.

\section{Activated Zeolite Preparation}

Natural zeolite rock was crushed and sieved to get particle size ranged from 8 to 10 mesh. Sieved zeolite was activated using $\mathrm{H}_{2} \mathrm{SO}_{4}$ solution with different 
Table 1. Activated carbon sample identification.

\begin{tabular}{cccc}
\hline ID & $\begin{array}{c}\text { Activation } \\
\text { method }\end{array}$ & $\begin{array}{c}\text { Activation } \\
\text { temperature }\left({ }^{\circ} \mathbf{C}\right)\end{array}$ & $\begin{array}{c}\text { Activation } \\
\text { time (hour) }\end{array}$ \\
\hline $700-1$ & physical & 700 & 1 \\
$750-1$ & physical & 750 & 1 \\
$750-2$ & physical & 750 & 2 \\
$850-1$ & physical & 850 & 1 \\
$900-1$ & physical & 900 & 1 \\
$900-2$ & physical & 900 & 2 \\
OH-700-1 & chemical & 700 & 1 \\
OH-750-1 & chemical & 750 & 1 \\
OH-750-2 & chemical & 750 & 2 \\
\hline
\end{tabular}

concentration i.e. $0.1,0.3,0.5$, and $1.0 \mathrm{M}$ inside a three necked flask equipped with heater and reflux apparatus (Wirawan, et al., 2013; Wirawan, et al., 2015). Typically $500 \mathrm{~g}$ natural zeolite was immersed into $800 \mathrm{ml} \mathrm{H}_{2} \mathrm{SO}_{4}$ solution inside the flask and then heated to $90^{\circ} \mathrm{C}$ and held for 1 to 2 hours. After cooled down, the activated zeolite was filtered and washed to normal $\mathrm{pH}$ and dried. The treated sample was identified as ZtMx with $\mathrm{t}$ is the time of activation and $x$ is the molar of the sulphuric acid solution, for example Z2M0.2 is the treated zeolite with $\mathrm{H}_{2} \mathrm{SO}_{4}$ $0.2 \mathrm{M}$ for 2 hours.

\section{Batch Adsorption}

Activated carbon and zeolite adsorption performance were tested using synthetic leaching solution of $\mathrm{CoSO}_{4}$ and $\mathrm{Li}_{2} \mathrm{SO}_{4}$ solution mixture with the final concentration of $100 \mathrm{ppm}$ for each metal. The batch adsorption method was done using $100 \mathrm{ml}$ capped Erlenmeyer flasks filled with $20 \mathrm{ml}$ cobalt and lithium solution mixture which were placed inside shaker waterbath. The ratio of the adsorbent over the synthetic solution was $10 \%$ or $20 \% \mathrm{w} / \mathrm{v}$ i.e. each volume of $20 \mathrm{ml}$ solution added 2 or 4 grams of the zeolite or carbon. Adsorption equilibrium time was fixed for 1 day, then, the solution was filtered and the filtrate was diluted 10 times for ICP analysis. Some kinetic adsorption experiments were also carried out by sampling the solution in a certain period of time.

\section{RESULTS AND DISCUSSION}

\section{Physical Activated Carbons Adsorption}

The single point batch adsorption test results for all physically activated char is shown in Figure 1.A. In general, the higher activation temperature will increase ions adsorption capacity. This is because the porosity development in the carbon will be much higher when activation temperature increase. It is interesting to note that for lower activation temperature the $\mathrm{Li}$ ions are adsorbed larger than Co. It can be the sign of microporosity 
94 The Development of Lithium Ion Recovery Method by Activated Carbon and Natural Zeolitebased Adsorbent
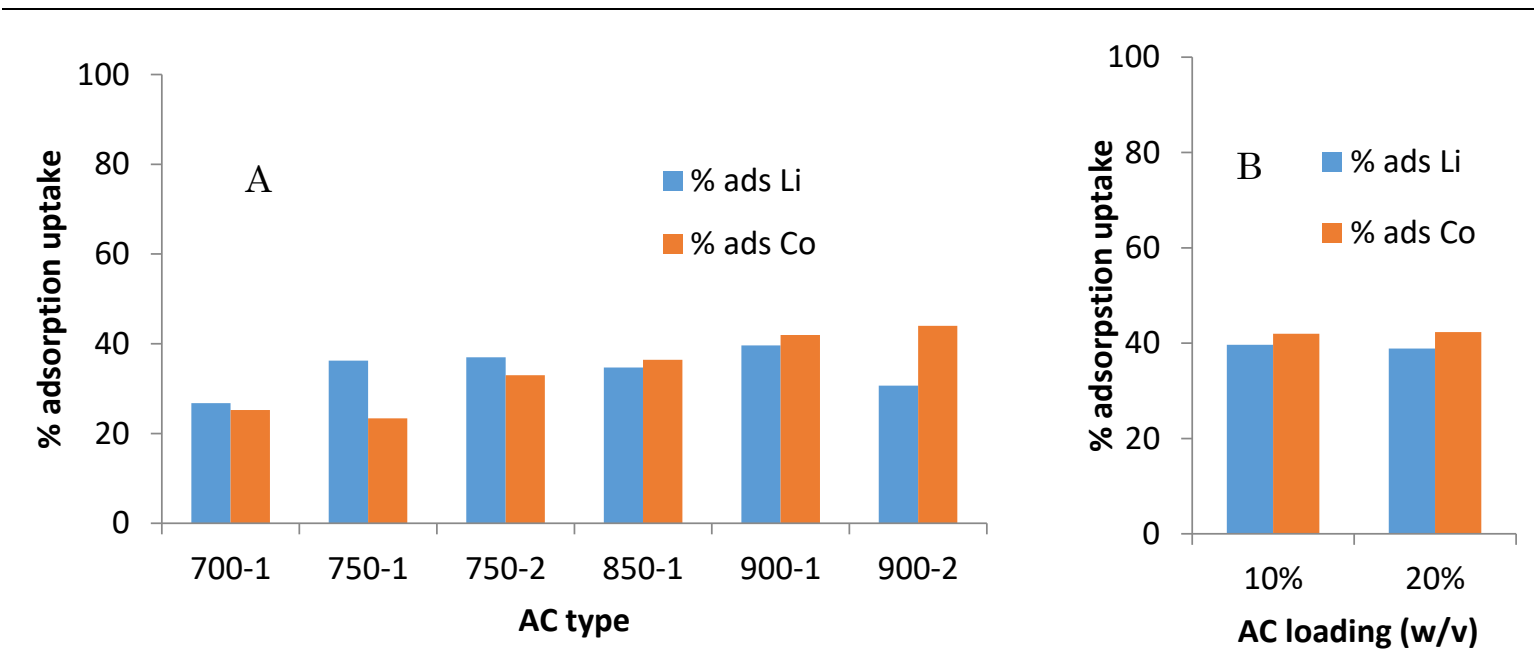

Fig.1: The adsorption capacity of activated carbon with different temperatures and activation time. B. Variation of ratio activated carbon:volume solution (w/v) using 900-1 sample for 2 hours.

domination of the activated char using this low temperature activation method. Cobalt ion size is much larger than $\mathrm{Li}$ ion, large size exclusion may apply in this micropore dominated adsorbent. When the temperature increased, the larger pore will be created which is favorable for larger particles to be adsorbed. In the same time, large pores will have lower attraction power to the small particles which may reduce the uptake capacity. This phenomenon is reflected in this adsorption experiment results as well, carbon with the activation temperature higher than $750 \mathrm{C}$ have better uptake capacity of Co than Li ions (Figure 1A).

Then, in terms of the length of activation time, the additional activation time from one to two hours increase the adsorption capacity of larger ions. Longer activation time will have the similar impact of increasing temperature which is pore enlargement. When the adsorbent content was increased to $20 \%$, there was no significant improvement both the adsorption capacity and the selectivity as shown in Figure 1.B. Thus, the adsorbent loading ratio of $10 \%$ is sufficient for this purpose.

From the results of the equilibrium adsorption approach above, the selectivity in separating $\mathrm{Li}$ and Co can be adjusted by varying temperature and time of activation. The results suggest for two separation scheme of $\mathrm{Li}$ ions, which are adsorbing as much as lithium using selective microporous adsorbent then the lithium can be obtained after leaching or adsorbing as much as possible other ions using large pore adsorbent then pure $\mathrm{Li}$ ions solution can be obtained.

The second method of removing ions other than lithium seems easier to be proceed. Li is very light component, it will be costly to develop a selective adsorbent of this ion. For further observation of the second recovery scheme, the kinetic mode of adsorption has been done using 900-2 carbon by observing the concentration of each element in a time interval during the 


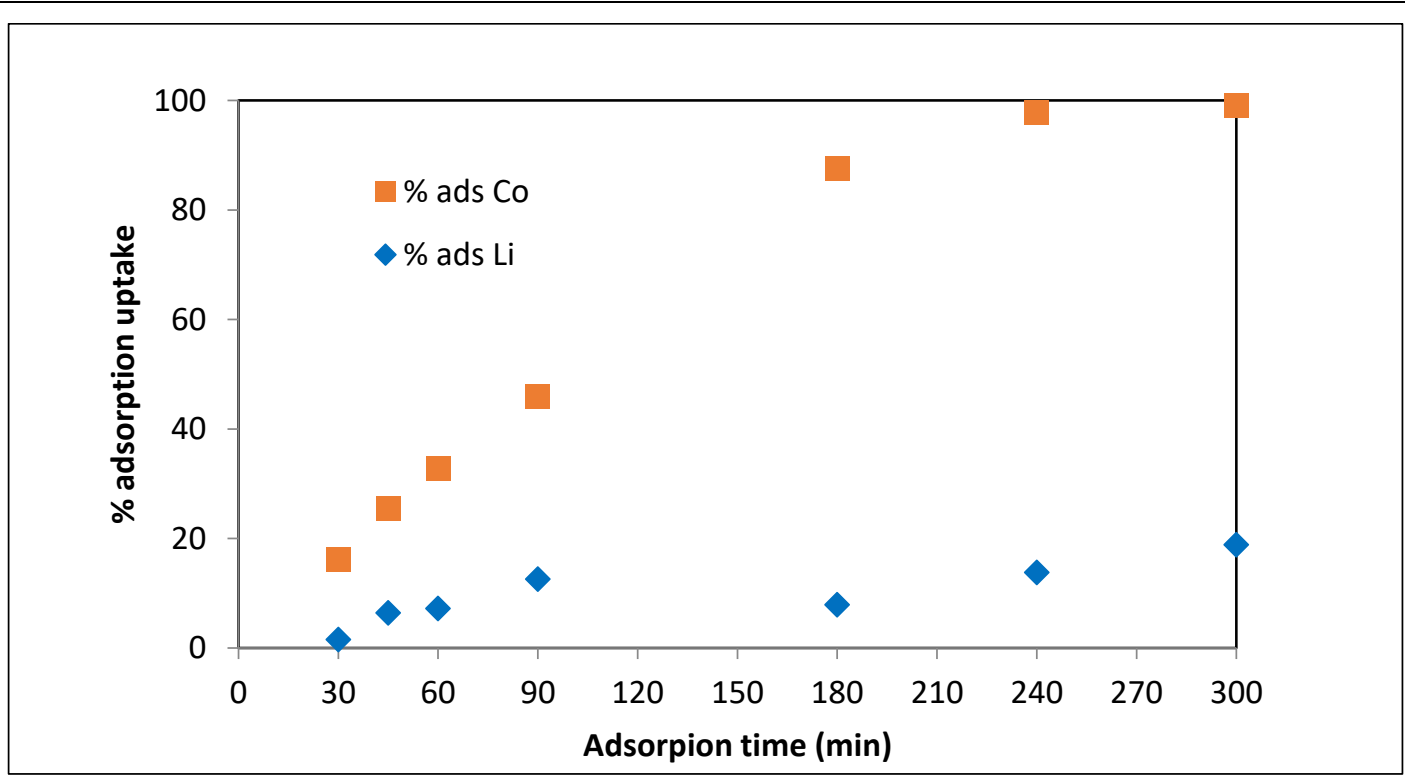

Fig.2: Adsorption kinetic of 900-2 sample to lithium and cobalt ion.

adsorption process. The concentration of each species was continuously monitored and the profile of increasing adsorption uptake for each ion was plotted in Figure 2. As expected, the adsorption profile shows that the longer the contact time, the adsorption uptake for all ions is getting higher, but for the selectivity between the ions has an optimum value due to the difference of adsorption rate. From the Figure 2, it can be seen that the optimum selectivity can be reach at about 180 minutes after the adsorption started and its getting lower by the progressing adsorption time because Co has been completely adsorbed while the $\mathrm{Li}$ ions adsorption are still progressing to the completion thus the gap of the ion uptake is getting closer. By this result, it is suggested to use 3 hours as the adsorption process optimum time to gain the best separation effect of the both ions which is the most lithium ions still in the solution while most of the cobalt ion are already adsorbed.

\section{Chemical Activated Carbon Adsorption}

As shown in Figure 3, the results of adsorption tests on activated carbon samples with chemical activation shows the higher adsorption capacity for higher activation temperature and the longer the time of activation samples. The adsorption selectivity is increasing by the increase of activation temperature but slightly decrease by increase the activation time to 2 hours. In the same activation temperature, the capacity of chemically activated carbon is always higher than the physically activated one. Thus the chemical agent enhances the porosity development of the parent carbon. The micropores are preserve during the longer activation period by using this activation scheme which is indicated by similar uptake of lithium ion of $\mathrm{OH}-750-1$ and $\mathrm{OH}-750-2$ samples. 
96 The Development of Lithium Ion Recovery Method by Activated Carbon and Natural Zeolitebased Adsorbent

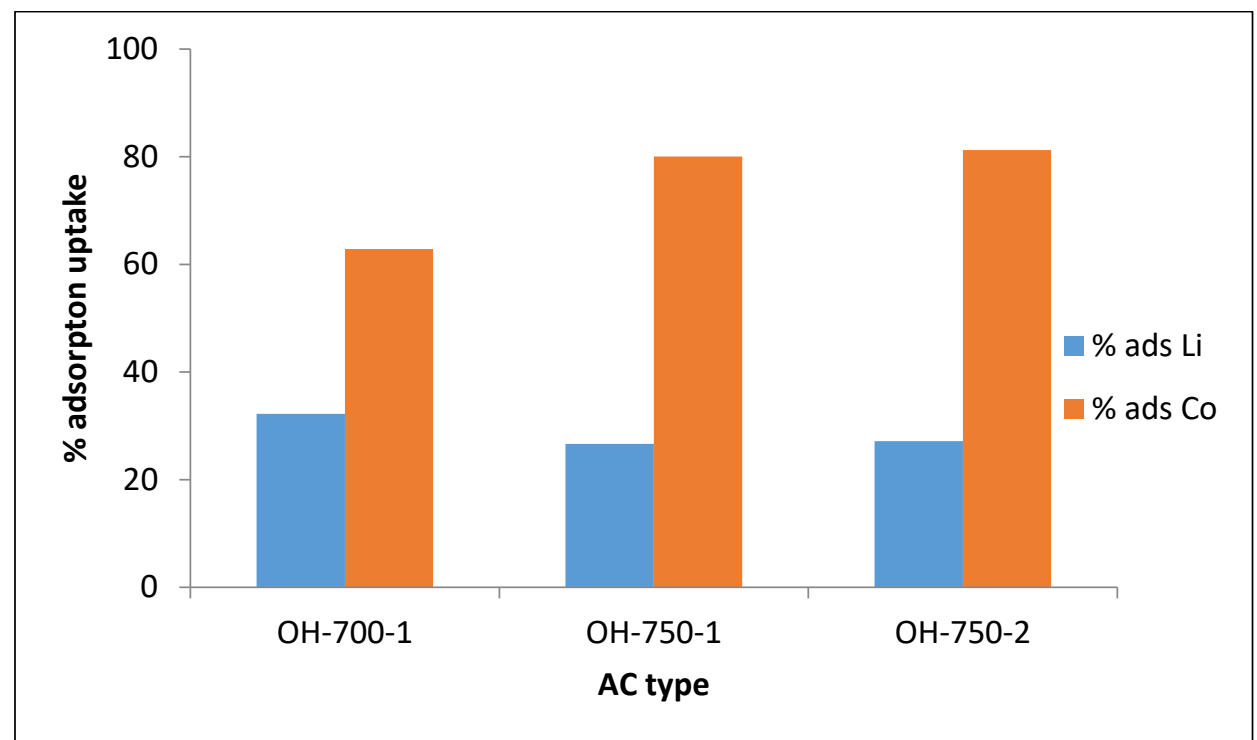

Fig.3: Adsorption capacity and selectivity of chemical and physical activated carbon samples in 2 hours adsorption.

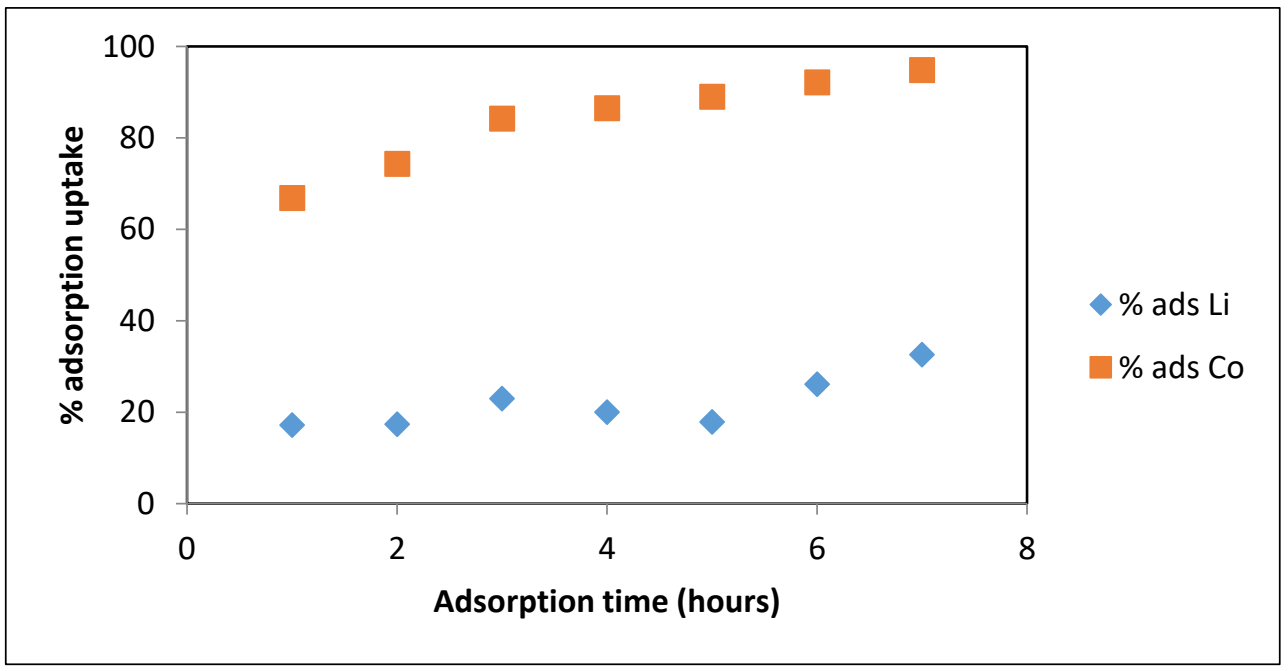

Fig.4: Adsorption kinetic of $\mathrm{OH}-750-1$ sample to lithium and cobalt ion.

Figure $\mathbf{4}$ is shown the data from kinetic adsorption using chemical activated sample. The test results of selective adsorption on activated carbon shows that the longer the contact time, the adsorption uptake capacity is getting higher. However, the optimum adsorption selectivity is at about 3 hours similar to the physically activated sample as discussed above. Further comparison among the two different activated samples, the $\mathrm{Li}$ ions adsorption uptake is increase faster using the chemically activated sample (Figure 4) than the physically activated sample (Figure 2). The fast uptake for the smaller particles could be due to abundantly available larger pore volume inside the $A C$ $(\mathrm{OH}-750-1)$. Large pores will have lower diffusion hindrance of particles to travel to the inner adsorption surface. 


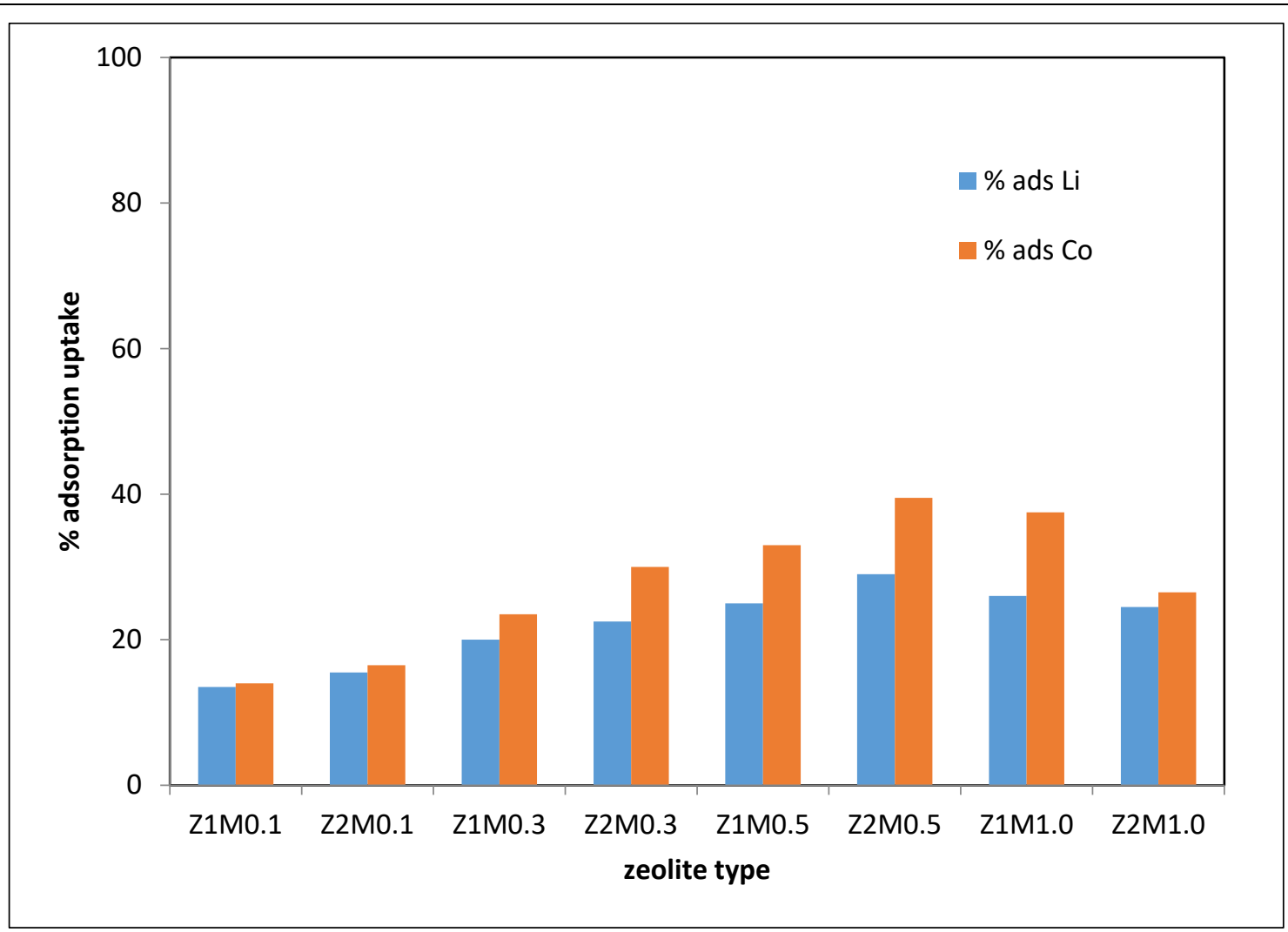

Fig.5: Adsorption capacity and selectivity of natural zeolite samples in 2 hour adsorption.

\section{Modified Zeolite Adsorption}

The drawback of using natural zeolites as the adsorbent of the mixture of cation is about the poor selectivity, since the zeolite will be able to exchange its naturally attached cations with all sort of cations in the solution. As shown in Figure 5 , most of the zeolites has similar uptake capacity for both the ions. The modification using acid solution will dilute some of $\mathrm{Al}$ content inside the material which alter the Si/Al ratio. The acidity and also crystal structure of natural zeolite will change by the modification of Si/Al ratio. In this study the selectivity of the uptake between the ions is increase by the acid modification. However, the harsh acid treatment may dilute a significant amount of alumina that can cause the collapse of zeolite crystal structure thus reducing the ion exchange capacity as indicated in Figure $\mathbf{5}$ of uptake depletion of the highly acidic modified samples.

\section{CONCLUSIONS}

It is shown that activated carbon from low cost coconut shell charcoal can be used for separating lithium from mixture solution. The proper activation can create porosity inside the char that suitable for take up other metal content while remaining most of the lithium on the solution. Meanwhile for natural zeolite, the most challenging task is to increase the selectivity of this material to be able to separate the lithium from the mixture. The acid modification may become an alternative in improving the selectivity of the natural zeolite. 
98 The Development of Lithium Ion Recovery Method by Activated Carbon and Natural Zeolitebased Adsorbent

\section{REFERENCES}

1. A. Tuncuk, V. Stazi, A. Akcil, E.Y. Yazici, $\mathrm{H}$. Deveci. "Aqueous metal recovery techniques from e-scrap: Hydrometalurgy in recycling." Minerals Engineering 25, 2012: 28-37.

2. Gulnaziya Issabayeva, Mohamed Kheireddine Aroua, Nik Meriam Sulaiman. "Continuous adsorption of lead ions in a column packed." Journal of Hazardous Materials 155 , 2008: 109-113.

3. Jia-Li Xiao, Shu-Ying Sun, Xingfu Song, Ping Li, Jian-Guo Yu. "Lithium ion recovery from brine using granulated polyacrylamide-MnO2." Chemical Engineering Journal, 279,, 2015: 659666.

4. Ji-Moon Jeong, Kyong Yop Rhee, SooJin Park. "Effect of chemical treatments on lithium recovery process of activated." Journal of Industrial and Engineering Chemistry, 27 „2015: 329333.

5. Ji-Moon Jeong, Kyong Yop Rhee, SooJin Park. "Effect of chemical treatments on lithium recovery process of activated." Journal of Industrial and Engineering Chemistry 27 , 2015: 329333.
6. Julien Lemaire a, Lenka Svecova, Fanny Lagallarde, Richard Laucournet, PierreXavier Thivel. "Lithium recovery from aqueous solution by sorption/desorption." Minerals Engineering, 25, 2012: 28-37.

7. K. Anoop Krishnan, T.S. Anirudhan. "Kinetic and equilibrium modelling of cobalt(II) adsorption." Chemical Engineering Journal, 137, 2008: 257264.

8. Zaira Zaman Chowdhury, Sharifuddin Mohd. Zain, Rashid Atta Khan, Rahman Faizur Farique. "Batch and fixed bed adsorption studies of lead (II) cations from aqueous solutions onto granular activated carbon derived from mangostana garcinia shell." Bioresources, 7, 3, 2012: 2895-2915.

9. Wirawan, S.K., Warmada, I.W., Wahyuni, E.T., 2013, ' Effect of ion exchanged to the $\mathrm{CO} 2$ adsorption on MFI zeolites', ASEAN Journal of Chemical Engineering, 13(2), 73-82

10. Wirawan, S.K., Sudibyo, H., Setiaji, M.F., Warmada, I.W., Wahyuni, E.T. , 2015 Development of natural zeolites adsorbent: Chemical analysis and preliminary TPD adsorption study, Journal of Engineering Science and Technology, 10 (spec. issued 4, 87-95 